\title{
Efeito da isquemia renal unilateral no rim contralateral avaliada pela expressão de Caspase 3
}

\author{
Effect of unilateral renal ischemia on the contralateral kidney assessed by \\ Caspase 3 expression
}

\begin{abstract}
Carolina Rodrigues Dal Bo 1,2 (D), Vitória Penido de Paula,2 (D), Anna Paula Weinhardt Baptista Strazzi (D), Nelson Wolosker ${ }^{1,2,4}$ (D), Thiago Pinheiro Arrais Aloia ${ }^{3,4}$ (D), Angela Mazzeo ${ }^{3}$ (D), Oskar Grau Kaufmann ${ }^{3,4}$ (D)
\end{abstract}

\begin{abstract}
Resumo
Contexto: Estudos demonstraram, por análise histológica e Dopplerfluxométrica, a interferência da isquemia renal unilateral, realizada em algumas cirurgias, sobre o rim contralateral, identificando o fenômeno de kidney-kidney crosstalk. Objetivos: Identificar o efeito da isquemia de duas estratégias de oclusão da vasculatura renal esquerda sobre o rim contralateral através do volume de células renais positivas para Caspase 3. Métodos: Suínos foram divididos em 2 grupos: $A(n=8)$, artéria renal esquerda clampeada, e AV $(n=8)$, artéria e veia renais esquerdas clampeadas. Foi realizado o estudo imuno-histoquímico (anti-Caspase 3), com o material de biópsias coletadas do rim isquêmico e contralateral em 0, 30, 60 e 90 minutos de isquemia, e análise morfométrica, sendo que a média representou o volume de área de Caspase 3 positiva (\%). Resultados: A análise morfométrica do rim contralateral nos tempos 30, 60 e 90 minutos de isquemia mostrou que a média da área marcada por Caspase 3 foi estatisticamente superior à média do rim isquêmico nos dois grupos: artéria renal clampeada (A) e artéria e veia renais clampeadas (AV). Comparando o rim isquêmico e contralateral nos dois tipos de clampeamento, não houve diferença estatisticamente significante da área marcada por Caspase 3. Conclusões: No modelo experimental de isquemia renal unilateral, o rim não isquêmico apresentou dano celular, demonstrado pela expressão da Caspase 3 de forma aguda em decorrência da isquemia contralateral. $O$ tipo de clampeamento do hilo não parece ter influência sobre o volume de área marcada por Caspase 3.
\end{abstract}

Palavras-chave: apoptose; rim; isquemia; imuno-histoquímica; Caspase 3.

\begin{abstract}
Background: Studies have demonstrated with histological analysis and Doppler flow measurement analysis that unilateral renal ischemia, which is performed in some surgeries, interfered with the contralateral kidney, identifying the phenomenon of kidney-kidney crosstalk. Objectives: To identify the effects on the ischemic and contralateral kidney of renal ischemia induced by two types of clamping technique by analyzing the volume of kidney cells positive for Caspase 3. Methods: Sixteen pigs were divided into 2 groups, as follows: A ( $n=8)$ - clamping of left renal artery only and AV ( $\mathrm{n}=8)$ - clamping of left renal artery and vein. Immunohistochemical analyses (anti Caspase 3 ) were conducted with biopsy specimens collected from the ischemic and contralateral kidney at 0, 30, 60, and 90 minutes of ischemia and morphometric analysis was performed, taking the mean to represent the volume of the Caspase 3 positive area (\%). Results: Morphometric analysis of specimens collected at 30, 60, and 90 minutes of ischemia showed that the mean area marked for Caspase 3 was statistically larger in the contralateral kidney than the ischemic kidney in both groups: clamped renal artery (A) and clamped renal artery and vein (AV). Comparing the ischemic and contralateral kidney, , there was no statistically significant difference in the area marked for Caspase 3 between the two types of clamping. Conclusions: In the experimental model of unilateral renal ischemia, the non-ischemic kidney exhibited cell damage, demonstrated by Caspase 3 expression. The type of hilum clamping does not appear to influence the area marked for Caspase 3.
\end{abstract}

Keywords: apoptosis; kidney; ischemia; immunohistochemistry; Caspase 3.

Como citar: Bo CRD, Paula VP, Strazzi APWB, et al. Efeito da isquemia renal unilateral no rim contralateral avaliada pela expressão de Caspase 3. J Vasc Bras. 2021;20:e20210040. https://doi.org/10.1590/1677-5449.210040

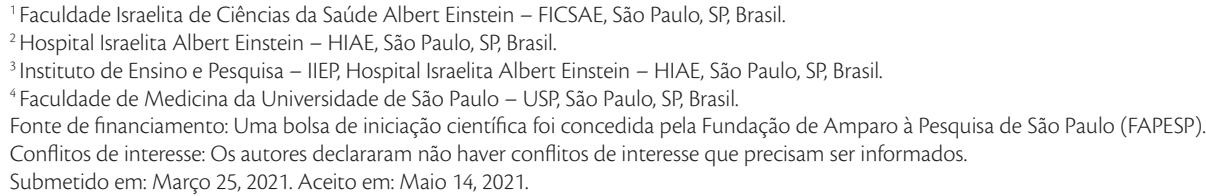




\section{INTRODUÇÃO}

A interrupção temporária do fluxo sanguíneo renal por meio do clampeamento da artéria renal ou do clampeamento simultâneo da artéria e da veia renais é um procedimento adotado rotineiramente na nefrectomia parcial a fim de reduzir sangramento intraoperatório ${ }^{1-4}$. Os pacientes submetidos a esse procedimento podem desenvolver insuficiência renal aguda transitória e até definitiva, e o tempo de clampeamento é um fator determinante.

A relação entre o risco de lesão renal e o tipo de clampeamento realizado é controverso na literatura alguns estudos indicam que a interrupção simultânea do fluxo arterial e venoso gera menos lesão renal após a cirurgia. Outros estudos sugerem que o clampeamento apenas da artéria renal seja uma melhor abordagem, justificando que a presença do fluxo venoso provoca menos danos ao rim ${ }^{5,6}$.

Diversos estudos experimentais ${ }^{7-9}$ demonstraram a interferência da isquemia renal na diminuição da função de órgãos à distância, como fígado, coração, cérebro e pulmão, em um mecanismo reconhecido como organ crosstalk. Dois estudos, recentemente, observaram a interferência da isquemia renal unilateral sobre o rim contralateral, identificando o fenômeno de kidney-kidney crosstalk, demonstrado por análise histológica e por aumento dos índices de resistência e pulsatilidade na análise Dopplerfluxométrica ${ }^{10,11}$.

A imuno-histoquímica foi demonstrada em estudos como uma técnica com alta sensibilidade para detectar precocemente lesões isquêmicas. Alguns autores sugerem o seu uso para complementar as lacunas da análise histológica na isquemia tecidual ${ }^{12-15}$. Entre os marcadores imuno-histoquímicos, a Caspase 3 é um marcador de apoptose conhecido e descrito em danos isquêmicos em vários órgãos, incluindo cérebro, coração e fígado ${ }^{16}$.

Após revisão da literatura, não identificamos estudos que avaliaram o efeito da isquemia renal unilateral sobre o rim contralateral através da análise imuno-histoquímica por Caspase 3.

\section{OBJETIVO}

O objetivo deste estudo foi identificar o efeito da isquemia renal por clampeamento simples da artéria renal esquerda e do clampeamento simultâneo da artéria e da veia renal esquerdas sobre o rim isquêmico e sobre o contralateral, analisando o volume de células renais positivas para Caspase 3 ao longo do tempo de isquemia.

\section{MÉTODOS}

O estudo operatório foi conduzido entre janeiro e dezembro de 2016 no Centro de Experimentação e Treinamento em Cirurgia do Hospital Israelita
Albert Einstein (CETEC), acreditado pela Associação Americana para Acreditação de Cuidados com Animais de Laboratório (AAALAC), estando de acordo com as normas expedidas pelo Conselho Nacional de Controle da Experimentação Animal (CONCEA) e tendo sido aprovado pelo Comitê de Uso e Cuidado de Animais do Hospital Israelita Albert Einstein (protocolo CEUA 3290). A análise imuno-histoquímica foi realizada no laboratório do Instituto Israelita de Ensino e Pesquisa Albert Einstein (IIEP) entre abril de 2018 e abril de 2019.

Dezesseis suínos da raça Large White, com idade entre 90 e 120 dias e pesando $30 \mathrm{~kg}$ cada, foram divididos em dois grupos de acordo com o tipo de clampeamento renal, contendo oito animais cada: o grupo AV, com clampeamento de artéria e veia renais esquerdas, e o grupo A, com clampeamento da artéria renal esquerda. Os animais foram submetidos a biópsias no rim isquêmico e no rim contralateral (rim direito - não isquêmico) concomitantemente nos tempos 0, 30, 60 e 90 minutos de isquemia. As biópsias foram processadas em blocos de parafina e fragmentadas em cortes de $5 \mu \mathrm{m}$ de espessura que, após serem fixados em lâminas, foram usados para análise imuno-histoquímica.

\section{Procedimento anestésico e técnica intraoperatória}

Todos os animais foram previamente anestesiados com injeção intramuscular de Cetamina $(10,0 \mathrm{mg} / \mathrm{kg})$ e Midazolam $(0,25 \mathrm{mg} / \mathrm{kg})$, misturados na mesma seringa. Passados 15 minutos da injeção, a veia marginal da orelha foi cateterizada com cateter de calibre 20 ou 22 (BD Insystem, BectonTherapy Systems Inc., Utah, EUA) ${ }^{17}$, para servir de acesso venoso para a indução anestésica, que foi realizada com Tiopental $7 \mathrm{mg} / \mathrm{kg}$. Para intubação dos animais, foram utilizados tubos endotraqueais tamanho 6,5 a 8,5 (Portex $^{\circledR}$, Minesota, EUA). A anestesia foi mantida inalatória com Isoflurano $2 \%$. A analgesia foi mantida com Fentanil, com dose inicial de $2,5 \mu \mathrm{g} / \mathrm{kg}^{18}$.

Com o animal anestesiado na posição de decúbito dorsal horizontal, realizou-se a higienização local e colocação dos campos cirúrgicos. Todos os animais foram submetidos à monitorização hemodinâmica com pressão arterial (PA) invasiva.

Efetuou-se laparotomia xifopúbica mediana e acesso ao retroperitônio com mobilização das alças. Após dissecção e reparo dos vasos renais, bilateralmente, registrou-se a aferição do diâmetro da artéria renal (valor médio de 3-4 mm) e o padrão do fluxo renal ao Doppler, no tempo zero, para descartar qualquer alteração prévia. Após a certificação de que ambos os rins eram morfologicamente adequados para análise, com volume máximo de $12 \mathrm{~cm}^{3}$, efetuou-se biópsia no tempo zero, em ambos os rins. 
Em seguida, foi realizado clampeamento do hilo renal esquerdo com clamp vascular tipo bulldog, e o direito permaneceu não isquêmico. A partir daí, foram realizadas biópsias seriadas do parênquima renal nos tempos 30, 60 e 90 minutos, em ambos os rins.

As biópsias foram padronizadas em $1 \times 1 \mathrm{~cm}^{2}$ na região cortical. As biópsias seriadas não afetaram negativamente os rins, pois foram realizadas no córtex em diferentes locais. Após 90 minutos, as pinças cirúrgicas foram retiradas, e, ao final do procedimento cirúrgico, os animais foram eutanasiados sob anestesia geral com overdose de Tiopental e cloreto de potássio $19,1 \%$ intravenoso (IV) (dose $15-30 \mathrm{mg} / \mathrm{kg}$ )

\section{Análise imuno-histoquímica}

Para a realização da análise imuno-histoquímica, as biópsias renais coletadas nos diferentes tempos foram fixadas em formol $10 \%$ por 24 horas, desidratados em soluções crescentes de álcool, diafanizados em xilol e incluídos em parafina. Foram utilizados quatro cortes de $5 \mu \mathrm{m}$ de espessura realizados em lâminas silanizadas para cada fragmento da biópsia/animal.

Após desparafinizadas, as lâminas foram hidratadas e colocadas em solução de ácido cítrico $10 \mathrm{mM}$ (pH 6,0), que foi aquecida por 12 minutos no micro-ondas para recuperação antigênica. Após resfriadas, as lâminas foram incubadas em solução de $\mathrm{H}_{2} \mathrm{O}_{2} 3 \%$ para bloqueio da peroxidase endógena. Posteriormente, as lâminas foram lavadas três vezes em solução salina tamponada (PBS) com sabão Tween $200,5 \%$ por 5 minutos e incubadas com um bloqueador de proteínas (LowProteinBlocking, eBioscience, ThermoFisherScientific Inc., Massachusetts, EUA). Após lavadas novamente em Tween 20, as lâminas foram incubadas overnight com anticorpo primário de coelho policlonal anti-Caspase 3 (ThermoFisherScientific Inc., Massachusetts, EUA).

No dia seguinte, as lâminas foram lavadas em Tween 20 e incubadas com anticorpo secundário de cabra anti-IGG $(\mathrm{H}+\mathrm{L})$ (ThermoFisherScientific Inc., Massachusetts, EUA). As lâminas foram reveladas por Diaminobenzidina-DAB (eBioscience ThermoFisherScientific Inc., Massachusetts, EUA) e contracoradas com hematoxilina por 1 minuto.

Finalmente, as lâminas foram desidratadas, imersas em xilol e protegidas por lamínulas para serem analisadas ao microscópio AxioVert A1 (Zeiss, Alemanha) e fotomicrografadas pela câmera digital Axio CAM 503 Color (Zeiss, Alemanha). Cada animal teve uma lâmina confeccionada por tempo $(0,30,60$ e 90 minutos) de cada rim (isquêmico e não isquêmico).

Foi realizada a análise morfométrica para calcular o volume de células positivas (\%) para Caspase 3 utilizando o software OLYMPUS cellSens Dimension (OLYMPUS CORPORATION, Tóquio, Japão).
Realizamos fotos de oito lâminas por animal. Cada lâmina continha quatro cortes do mesmo bloco parafinado da biópsia, sendo o primeiro corte o controle negativo da reação. Foram realizadas fotos de dez campos aleatórios, sendo que a média representou o volume de área de Caspase 3 positiva expressa em porcentagem por animal.

\section{Análise estatística}

A realização desta pesquisa experimental gerou dados de forma controlada, prospectiva e randomizada. Os dados foram analisados estatisticamente usado o pacote estatístico R (The R Foundation, Viena, Áustria) ${ }^{19}$. As medidas avaliadas são todas quantitativas e foram descritas como médias e desvio padrão. O nível de significância adotado foi de 5\%.

Considerando a variabilidade baixa entre animais e respeitando o princípio bioético dos 3 Rs em estudo experimentais com animais (substituição, refinamento e redução), para definição do número amostral, realizamos um estudo com 16 suínos, e, a partir de dados preliminares, as variáveis foram avaliadas em relação à sua distribuição por meio dos gráficos de quantis, histograma e boxplot, além do teste de Shapiro-Wilk. Foi observado que a maior parte dos dados se concentrava próxima da média, e a variação dos dados em relação à média era pequena, não sendo rejeitada a suposição de normalidade. Isso sugere que a amostra representava bem a população estudada e era suficiente para aplicar testes estatísticos com segurança, não sendo necessário submeter mais animais ao estudo experimental.

A comparação entre as áreas marcadas nos grupos definidos a partir do tipo de clampeamento (A, artéria renal unilateral, e AV, artéria e veia renais unilaterais) e lado do rim (direito, não isquêmico, e esquerdo, isquêmico) foi feita por meio de modelos de regressão lineares mistos a fim de contemplar a dependência entre as diferentes medidas realizadas em um mesmo animal ${ }^{19-21}$.

\section{RESULTADOS}

No rim isquêmico e no rim contralateral, a média estimada da área marcada por Caspase 3 no grupo A (clampeamento unilateral da artéria renal) não foi estatisticamente diferente da média do grupo AV (clampeamento unilateral da artéria e veia renais) em todos os momentos (Tabelas 1 e 2). No grupo em que foi realizado clampeamento unilateral da artéria e veia renal (grupo AV), a média estimada da área marcada por Caspase 3 no rim não isquêmico foi estatisticamente superior à média do rim isquêmico em todos os instantes de tempo, exceto no momento inicial (tempo zero) (Tabela 3 e Figura 1). 
Tabela 1. Área marcada por Caspase 3 no rim isquêmico pelos grupos A e AV e resultado do modelo de regressão linear misto para a área marcada como desfecho.

\begin{tabular}{cccc}
\hline Tempo de isquemia & A (\%) & AV (\%) & DM (IC 95\%) \\
\hline 0 minuto & $0,02(0,02)$ & $0,02(0,04)$ & $0,00(-0,02 ; 0,02)$ \\
$\mathbf{3 0}$ minutos & $0,24(0,20)$ & $0,20(0,16)$ & $-0,04(-0,23 ; 0,15)$ \\
$\mathbf{6 0}$ minutos & $0,11(0,13)$ & $0,13(0,12)$ & $0,02(-0,15 ; 0,18)$ \\
$\mathbf{9 0}$ minutos & $0,55(0,36)$ & $0,70(0,34)$ & $0,15(-0,20 ; 0,51)$ \\
\hline
\end{tabular}

$\mathrm{DM}$ = diferença de médias entre os grupos $\mathrm{A}$ e $\mathrm{AV}$ no rim isquêmico; $\mathrm{IC}$ = intervalo de confiança; Valor-p referente ao modelo de regressão linear misto; Desvio padrão em parênteses.

Tabela 2. Média do volume de área marcada por Caspase 3 no rim contralateral (não isquêmico) pelos grupos A e AV e resultado do modelo de regressão linear misto para área marcada como desfecho.

\begin{tabular}{ccccc}
\hline Tempo de isquemia & $\mathrm{A}(\%)$ & $\mathrm{AV}(\%)$ & $\mathrm{DM}(\mathrm{IC} 95 \%)$ & Valor $\mathrm{p}$ \\
\hline $\mathbf{0}$ minuto & $0,00(0,00)$ & $0,03(0,05)$ & $0,03(-0,01 ; 0,06)$ & 0,146 \\
$\mathbf{3 0}$ minutos & $1,01(0,35)$ & $1,11(0,50)$ & $0,11(-0,25 ; 0,46)$ & 0,528 \\
$\mathbf{6 0}$ minutos & $1,24(0,79)$ & $1,24(0,71)$ & $-0,01(-0,68 ; 0,65)$ & 0,965 \\
$\mathbf{9 0}$ minutos & $0,84(0,57)$ & $1,11(0,49)$ & $0,29(-0,21 ; 0,79)$ & 0,229 \\
\hline
\end{tabular}

$\mathrm{DM}=$ diferença de médias entre os grupos $\mathrm{A}$ e $\mathrm{AV}$ no rim não isquêmico; $\mathrm{IC}$ = intervalo de confiança; Valor-p referente ao modelo de regressão linear misto; Desvio padrão em parênteses.

Tabela 3. Média do volume de área marcada por Caspase 3 no grupo AV no rim isquêmico e no não isquêmico e resultado do modelo de regressão linear misto para área marcada como desfecho.

\begin{tabular}{ccccr}
\hline Tempo de isquemia & Rim não isquêmico (\%) & Rim isquêmico (\%) & DM (IC 95\%) & Valor p \\
\hline O minuto & $0,03(0,05)$ & $0,02(0,04)$ & $-0,01(-0,02 ; 0,00)$ & 0,135 \\
$\mathbf{3 0}$ minutos & $1,11(0,50)$ & $0,20(0,16)$ & $-1,03(-1,14 ;-0,91)$ & $<0,001$ \\
$\mathbf{6 0}$ minutos & $1,24(0,71)$ & $0,13(0,12)$ & $-1,15(-1,33 ;-0,98)$ & $<0,001$ \\
$\mathbf{9 0}$ minutos & $1,11(0,49)$ & $0,70(0,34)$ & $-0,41(-0,56 ;-0,26)$ & $<0,001$ \\
\hline
\end{tabular}

$\mathrm{DM}=$ diferença de médias entre o rim isquêmico e não isquêmico no grupo $\mathrm{AV} ; \mathrm{IC}=$ intervalo de confiança; Valor-p referente ao modelo de regressão linear misto; Desvio padrão em parênteses.

No grupo em que foi realizado clampeamento unilateral da artéria renal (grupo A), a média estimada da área marcada por Caspase 3 do rim não isquêmico foi maior que a média do rim isquêmico em todos os instantes de tempo, sendo essa diferença estatisticamente significante (Tabela 4).

\section{DISCUSSÃO}

Nos animais submetidos ao clampeamento apenas da artéria renal, houve diferença estatisticamente significante no tempo zero entre o rim isquêmico e o não isquêmico. Apesar disso, ao longo do tempo (30, 60 e 90 minutos), o rim não isquêmico apresentou uma progressão de lesão significativa, o que indica que as consequências da isquemia renal são refletidas no rim não isquêmico.

Nos animais submetidos ao clampeamento simultâneo da artéria e da veia renal, no tempo inicial não havia diferença estatisticamente significante na área marcada por Caspase 3 entre o rim isquêmico e o rim não isquêmico. Com o passar do tempo, observou-se aumento da área marcada por Caspase 3 no rim não isquêmico, o que pode indicar que a apoptose celular sofrida pelo rim isquêmico também seja refletida no rim contralateral.

A lesão isquêmica do rim geralmente resulta em danos às células do néfron e às células da vasculatura renal. Essas células são perdidas através do processo de necrose e apoptose, podendo levar à falência renal ${ }^{22}$. A isquemia causada por uma obstrução nas artérias do rim prejudica a chegada de nutrientes e de oxigênio, alterando a respiração celular e, consequentemente, 0 metabolismo renal através de uma cascata de reações bioquímicas decorrentes da falta de energia (ATP) ${ }^{23}$. Outros danos também são verificados, tais como alteração no citoesqueleto celular, na polaridade da superfície das membranas, na ativação de fosfolipases e proteases e, por fim, na incapacidade das funções celulares dependentes da enzima ATPase (bomba de sódio-potássio $)^{24}$.

Após a finalização da manobra cirúrgica de clampeamento, o fluxo sanguíneo é restabelecido, e a reperfusão renal, nesse cenário, intensifica as lesões causadas pela isquemia através da produção de espécies reativas de oxigênio, além da ocorrência de desarranjo celular, hipercoagulabilidade e congestão da microcirculação. Isso favorece a redução do fluxo 
Tabela 4. Média do volume de área marcada por Caspase 3 no grupo A no rim isquêmico e no não isquêmico e resultado do modelo de regressão linear misto para área marcada como desfecho.

\begin{tabular}{ccccc}
\hline Tempo de isquemia & Rim não isquêmico (\%) & Rim isquêmico (\%) & DM (IC 95\%) & Valor $p$ \\
\hline $\mathbf{0}$ minuto & $0,00(0,00)$ & $0,02(0,02)$ & $0,02(0,01 ; 0,02)$ & $<0,001$ \\
$\mathbf{3 0}$ minutos & $1,01(0,35)$ & $0,24(0,20)$ & $-0,79(-0,90 ;-0,69)$ & $<0,001$ \\
$\mathbf{6}$ minutos & $1,24(0,79)$ & $0,11(0,13)$ & $-1,29(-1,48 ;-1,11)$ & $<0,001$ \\
$\mathbf{9}$ minutos & $0,84(0,57)$ & $0,55(0,36)$ & $-0,27(-0,42 ;-0,13)$ & $<0,001$ \\
\hline
\end{tabular}

$\mathrm{DM}=$ diferença de médias entre o rim isquêmico e não isquêmico no grupo $\mathrm{A}$; IC = intervalo de confiança; Valor-p referente ao modelo de regressão linear misto; Desvio padrão em parênteses.

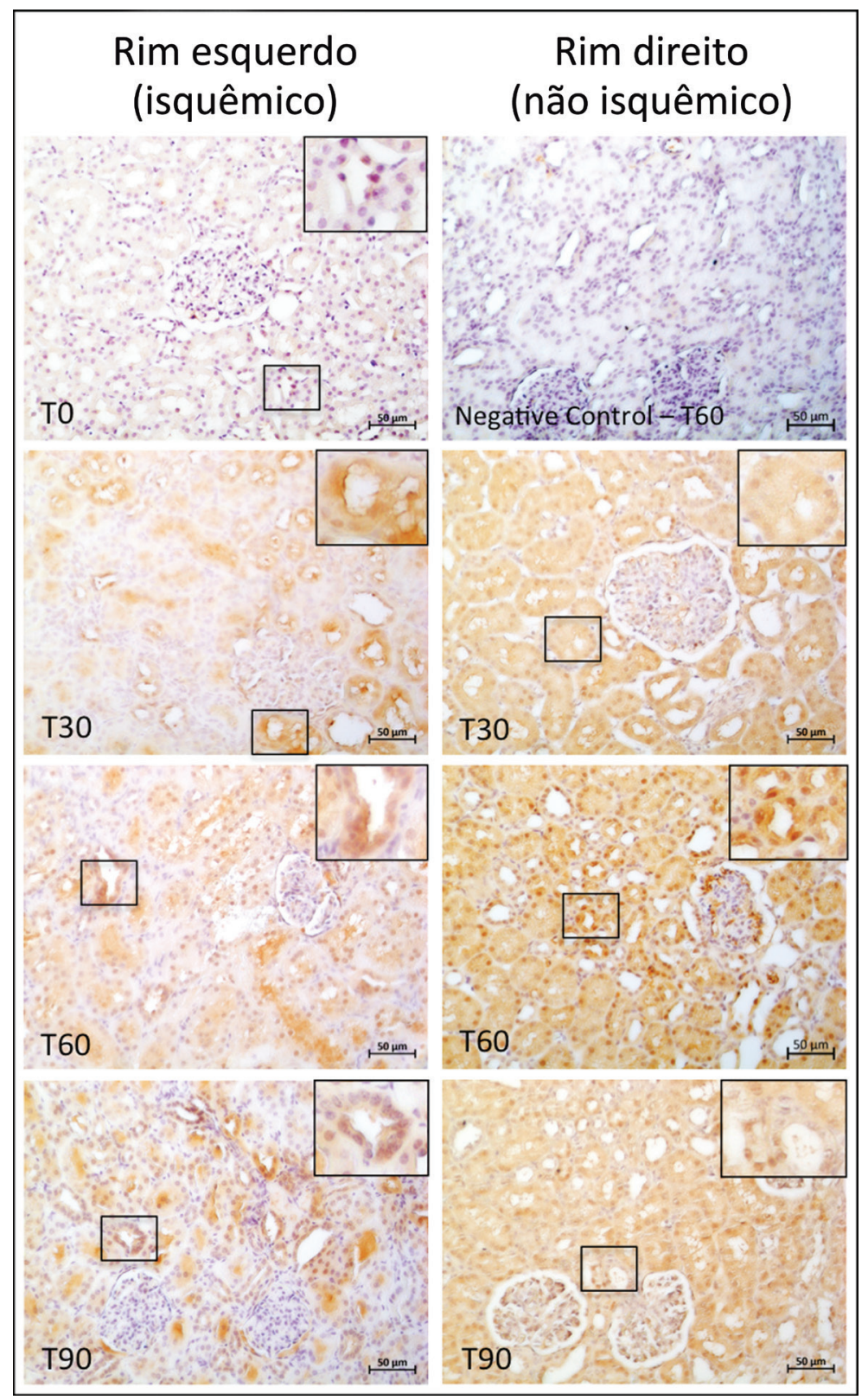

Figura 1. Imunomarcação por Caspase 3 nos túbulos contorcidos renais representados pela cor marrom-acastanhado. A marcação é citoplasmática e nuclear, e as imagens comparam o rim isquêmico (rim esquerdo) com o rim não isquêmico (rim direito) nos tempos 0, 30, 60 e 90 de isquemia. (T0) Rim esquerdo sem clamp (controle positivo); (Negative control - T60) Controle negativo da reação; (T30) 30 minutos de isquemia renal unilateral; (T60) 60 minutos de isquemia renal unilateral; (T90) 90 minutos de isquemia renal unilateral; Barra: $50 \mu \mathrm{m}$. 
sanguíneo renal, o que caracteriza o quadro clínico da insuficiência renal ${ }^{25}$. Nesse sentido, levando em consideração que, neste estudo, o rim não isquêmico sofreu maior apoptose que o rim isquêmico, uma explicação seria que a isquemia pode estar momentaneamente protegendo o rim em que o hilo renal está clampeado.

O fenômeno organ crosstalk pode ser definido como a comunicação biológica mútua entre órgãos distantes mediada por fatores de sinalização. Esse fenômeno ajuda a coordenar e manter a homeostase, mas uma súbita disfunção em qualquer órgão causa desregulação em outro órgão ${ }^{26}$.

Essa interferência parece estar envolvida com a cascata inflamatória gerada pelo mecanismo de isquemia. Entretanto, até onde sabemos, não há estudos experimentais que comprovem a interferência com imunomarcadores do rim isquêmico na função do rim contralateral, não isquêmico.

Um estudo que realizou análise histológica de rins de suínos para estudar o fenômeno kidney-kidney crosstalk demonstrou aumento significativo $(\mathrm{p}<0,05)$ do número de lesões no rim controle a partir de 10-20 minutos de isquemia clampeando apenas a artéria renal e somente com 50-60 minutos de isquemia clampeando artéria e veia renais ${ }^{10}$.

No entanto, em relação à apoptose, o presente estudo não identificou diferença estatisticamente significante na área marcada por Caspase 3 entre o grupo em que foi clampeada a artéria renal exclusivamente e o grupo em que foi clampeada tanto a artéria quanto a veia renais.

Em relação às limitações, este estudo foi experimental, delineado com suínos, pois eles são excelentes modelos biomédicos dadas as semelhanças anatômicas, fisiológicas e imunológicas ao ser humano. Entretanto, os rins desses animais possuem uma maior tolerância ao tempo de isquemia do que o rim humano ${ }^{27}$.

A isquemia renal unilateral foi realizada até 90 minutos em uma tentativa de identificar qualquer diferença possível entre os dois tipos de clampeamento (apenas artéria renal vs. artéria e veia renais). Após 120 minutos de cirurgia, em média, a maioria dos animais encontrava-se em estado hipotensivo e sem resposta em decorrência de desidratação, hipotermia e, possivelmente, intolerância anestésica.

Devido à dificuldade em manter os animais vivos, tivemos uma amostra insuficiente para avaliação da segurança do efeito da reperfusão renal no rim contralateral, a qual deve ser considerada em investigações futuras.

\section{CONCLUSÃO}

No modelo experimental de isquemia renal unilateral, o rim não isquêmico apresentou dano celular, demonstrado pela expressão da Caspase
3 , de forma aguda em decorrência da isquemia contralateral. O tipo de clampeamento do hilo (artéria renal unilateral vs. artéria e veia renais unilaterais) não parece ter influência sobre o volume de área marcada por Caspase 3.

\section{REFERÊNCIAS}

1. Abuelo JG. Normotensive ischemic acute renal failure. N Engl J Med. 2007;357(8):797-805. http://dx.doi.org/10.1056/NEJMra064398. PMid:17715412.

2. Badr KF, Ichikawa I. Prerenal failure: a deleterious shift from renal compensation to decompensation. N Engl J Med. 1988;319(10):623-9. http://dx.doi.org/10.1056/NEJM198809083191007. PMid:3045546.

3. Simmons $M N$, Schreiber MJ, Gill IS. Surgical renal ischemia: a contemporary overview. J Urol. 2008;180(1):19-30. http://dx.doi. org/10.1016/j.juro.2008.03.022. PMid:18485395.

4. Himmelfarb J, Joannidis $M$, Molitoris B, et al. Evaluation and initial management of acute kidney injury. Clin J Am Soc Nephrol. 2008;3(4):962-7. http://dx.doi.org/10.2215/CJN.04971107. PMid:18354074.

5. Orvieto MA, Zorn KC, Mendiola F, et al. Recovery of renal function after complete renal hilar versus artery alone clamping during open and laparoscopic surgery. J Urol. 2007;177(6):2371-4. http:// dx.doi.org/10.1016/j.juro.2007.01.115. PMid:17509361.

6. Kerbl K, Chandhoke PS, Clayman RV, McDougall E, Stone AM, Figenshau RS. Ligation of the renal pedicle during laparoscopic nephrectomy: a comparison of staples, clips, and sutures. J Laparoendosc Surg. 1993;3(1):9-12. http://dx.doi.org/10.1089/ Ips.1993.3.9. PMid:8453134.

7. Gardner DS, De Brot S, Dunford LJ, et al. Remote effects of acute kidney injury in a porcine model. Am J Physiol Renal Physiol. 2016;310(4):F259-71. http://dx.doi.org/10.1152/ajprenal.00389.2015. PMid:26608790.

8. Crane NJ, Huffman SW, Alemozaffar M, Gage FA, Levin IW, Elster EA. Evidence of a heterogeneous tissue oxygenation: renal ischemia/reperfusion injury in a large animal model. J Biomed Opt. 2013;18(3):035001. http://dx.doi.org/10.1117/1.JBO.18.3.035001. PMid:23456040.

9. Oishi Y, Manabe I. Organ system crosstalk in cardiometabolic disease in the age of multimorbidity. Front Cardiovasc Med. 2020;7:64. http://dx.doi.org/10.3389/fcvm.2020.00064.

10. Baptista Sincos AP, Mazzeo A, Sincos IR, et al. Duplex scan and histologic assessment of acute renal injury in a kidney-kidney crosstalk swine experimental model. J Vasc Surg. 2018;68(2):58895. http://dx.doi.org/10.1016/j.jvs.2017.06.118. PMid:28958477.

11. Mazzeo A, Sincos APB, Leite KRM, Goes MAJr, Pavão OFS, Kaufmann OG. Study of kidney morphologic and structural changes related to different ischemia times and types of clamping of the renal vascular pedicle. Int Braz J Urol. 2019;45(4):754-62. http://dx.doi. org/10.1590/s1677-5538.ibju.2018.0559. PMid:31184454.

12. Yanagihara T, Yoshimine $T$, Morimoto $K$, Yamamoto $K$, Homburger HA. Immunohistochemical Investigation of Cerebral Ischemia in Gerbils. J Neuropathol Exp Neurol. 1985;44(2):204-15. http:// dx.doi.org/10.1097/00005072-198503000-00009. PMid:2579210.

13. Mondello C, Cardia L, Ventura-Spagnolo E. Immunohistochemical detection of early myocardial infarction: a systematic review. Int J Legal Med. 2017;131(2):411-21. http://dx.doi.org/10.1007/ s00414-016-1494-1. PMid:27885432.

14. Aljakna A, Fracasso T, Sabatasso S. Molecular tissue changes in early myocardial ischemia: from pathophysiology to the identification 
of new diagnostic markers. Int J Legal Med. 2018;132(2):425-38. http://dx.doi.org/10.1007/s00414-017-1750-z. PMid:29362873.

15. Mondello C, Cardia L, Bartoloni G, Asmundo A, Ventura Spagnolo E. Immunohistochemical study on dystrophin expression in CAD-related sudden cardiac death: a marker of early myocardial ischaemia. Int J Legal Med. 2018;132(5):1333-9. http://dx.doi. org/10.1007/s00414-018-1843-3. PMid:29732464.

16. Faubel S, Edelstein CL. Caspases as drug targets in ischemic organ injury. Curr Drug Target Immune Endocr Metabol Disord. 2005;5(3):269-87. http://dx.doi.org/10.2174/1568008054863754. PMid: 16178788.

17. Belczak SQ. Tratamento endovascular de trauma arterial periférico com o uso de stents revestidos: estudo experimental em porcos [tese]. São Paulo: Faculdade de Medicina, Universidade de São Paulo; 2011.

18. Lederman A. Indução de aneurisma em aorta abdominal de porcos: um modelo endovascular [tese]. São Paulo: Faculdade de Medicina, Universidade de São Paulo; 2015.

19. R Core Team. R: A language and environment for statistical computing [software]. New Zeland: R Foundation for Statistical Computing; 2017. [cited 2021 jan 18]. https://www.R-project.org/.

20. Morettin PA, Bussab WO. Medidas-Resumo. In: Morettin PA, Bussab WO, editors. Estatística básica. São Paulo: Saraiva; 2010. p. 35-56.

21. Faraway JJ. Multinomial Data. In: Faraway JJ, editor. Extending the linear model with R: generalized linear, mixed effects and nonparametric regression models. Boca Raton: Chapman \& Hall/ CRC; 2016. p. 129-50. http://dx.doi.org/10.1201/9781315382722-9.

22. BonventreJV, Weinberg JM. Recent advances in the pathophysiology of ischemic acute renal failure. J Am Soc Nephrol. 2003;14(8):2199210. http://dx.doi.org/10.1097/01.ASN.0000079785.13922.F6. PMid: 12874476 .

23. Secin FP. Importance and limits of ischemia in renal partial surgery: experimental and clinical research. Adv Urol. 2008;2008:102461. http://dx.doi.org/10.1155/2008/102461. PMid:18645616.

24. Goes N, Urmson J, Ramassar V, Halloran PF. Ischemic acute tubular necrosis induces an extensive local cytokine response. Evidence for induction of interferon-gamma, transforming growth factor-beta 1 , granulocyte-macrophage colony-stimulating factor, interleukin-2, and interleukin-10. Transplantation. 1995;59(4):565-72. http:// dx.doi.org/10.1097/00007890-199502270-00022. PMid:7878762.

25. Jablonski P, Howden B, Rae D, et al. The influence of the contralatera kidney upon recovery from unilateral warm renal ischemia. Pathology. 1985;17(4):623-7. http://dx.doi.org/10.3109/00313028509084764. PMid:4094791.

26. Armutcu F. Organ crosstalk: the potent roles of inflammation and fibrotic changes in the course of organ interactions. Inflamm Res. 2019;68(10):825-39. http://dx.doi.org/10.1007/s00011-019-01271-7. PMid:31327029.

27. Giraud S, Favreau F, Chatauret N, Thuillier R, Maiga S, Hauet $\mathrm{T}$. Contribution of large pig for renal ischemia-reperfusion and transplantation studies: the preclinical model.J Biomed Biotechnol. 2011;532127. http://dx.doi.org/10.1155/2011/532127.

Correspondência Carolina Rodrigues Dal Bo Hospital Israelita Albert Einstein - HIAE Avenida Albert Einstein, 627 - Morumbi 05652-900 - São Paulo (SP), Brasil Tel.: (11) 2151-0011 E-mail: carolinardalbo@gmail.com

Informações sobre os autores CRDB e VPP - Acadêmicas, $6^{\circ}$ ano, Medicina, Faculdade Israelita de Ciências da Saúde Albert Einstein (FICSAE); Alunas de Iniciação Científica, Grupo de Pesquisa em Cirurgia Vascular, Hospital Israelita Albert Einstein (HIAE).

APWBS - Cirurgiã Vascular, Especialista, Sociedade Brasileira de Angiologia e Cirurgia Vascular (SBACV), Doutorado em Ciências da

Saúde; Pós-Doutoramento em Cirurgia Endovascular, Instituto de Ensino e Pesquisa (IIEP), Hospital Israelita Albert Einstein (HIAE). NW - Cirurgião Vascular, Vice-presidente de Pesquisa e Inovação, Hospital Israelita Albert Einstein (HIAE); Doutorado em Ciências da Saúde, Universidade de São Paulo (USP); Docente, Programa de Pós-graduação stricto sensu de Ciências da Saúde, Faculdade Israelita Albert Finstein (FICSAE), Professor Livre-docente e Associado, Faculdade de Medicina, Universidade de São Paulo (USP); Pesquisador nível I CNPq. TPAA - Biólogo, Pontifícia Universidade Católica de Goiás (PUC-GO); Especialista em Anatomia Comparada, Biologia do Desenvolvimento e Células Tronco; Mestrado e Doutorado em Anatomia dos Animais Domésticos e Silvestres, Universidade de São Paulo (USP); Pós-

Doutorado em Neuroimunomodulação, Universidade de São Paulo (USP), Hepatologia Experimental, Instituto de Ensino e Pesquisa (IIEP), Hospital Israelita Albert Einstein (HIAE).

AM - Farmacêutica, Mestrado, Instituto de Ensino e Pesquisa (IIEP), Hospital Israelita Albert Einstein (HIAE); Especializada MBA, Gestão de Projetos, Universidade São Judas Tadeu (USTJ).

OGK - Urologista, Doutorado em Urologia, Universidade de São

Paulo (USP), Pós-doutorado em Cirurgia Robótica, Laparoscopia e Endourologia, Universidade da California, Irvine; MBA Executivo,

Gestão de Saúde Einstein/Insper; Docente Colaborador, Pósgraduação, Instituto de Ensino e Pesquisa (IIEP), Hospital Israelita Albert Einstein (HIAE).

Contribuição dos autores Concepção e desenho do estudo: CRDB, VPP, APWBS, TPAA, NW, OGK Análise e interpretação dos dados: CRDB, VPP, APWBS, TPAA Coleta de dados: CRDB, VPP, APWBS, AM Redação do artigo: CRDB, VPP, APWBS, TPAA Revisão crítica do texto: CRDB, VPP, APWBS, TPAA, NW, OGK Aprovação final do artigo*: CRDB, VPP, APWBS, AM, TPAA, NW,

OGK

Análise estatística: CRDB, VPP, OGK Responsabilidade geral pelo estudo: APWBS, NW, OGK

*Todos os autores leram e aprovaram a versão final submetida ao J Vasc Bras. 